TO APpear in the Astrophysical Journal

Preprint typeset using IATEX style emulateapj v. 11/12/01

\title{
A NEW CLUE TO THE TRANSITION MECHANISM BETWEEN OPTICAL HIGH AND LOW STATES OF THE SUPERSOFT X-RAY SOURCE RX J0513.9-6951, IMPLIED FROM THE RECURRENT NOVA CI AQUILAE 2000 OUTBURST MODEL
}

\author{
IZUMI HACHISU \\ Department of Earth Science and Astronomy, College of Arts and Sciences, University of Tokyo, Komaba, \\ Meguro-ku, Tokyo 153-8902, Japan \\ hachisu@chianti.c.u-tokyo.ac.jp \\ AND \\ MARIKO KATO \\ Department of Astronomy, Keio University, Hiyoshi, Kouhoku-ku, Yokohama 223-8521, Japan \\ mariko@educ.cc.keio.ac.jp \\ to appear in the Astrophysical Journal
}

\begin{abstract}
We have found a new clue to the transition mechanism between optical high/X-ray off and optical low/X-ray on states of the LMC supersoft X-ray source RX J0513.9-6951. A sharp $~ 1$ mag drop is common to the CI Aql 2000 outburst. These drops are naturally attributed to cessation of optically thick winds on white dwarfs. A detailed light-curve analysis of CI Aql indicates that the size of a disk drastically shrinks when the wind stops. This causes $\sim 1-2$ mag drop in the optical light curve. In RX J0513.9-6951, the same mechanism reproduces sharp $\sim 1$ mag drop from optical high to low states. We predict this mechanism also works on the transition from low to high states. Interaction between the wind and the companion star attenuates the mass transfer and drives full cycles of low and high states.
\end{abstract}

Subject headings: binaries: close - novae, cataclysmic variables — stars: individual (CI Aquilae, RX J0513.9-6951) — stars: winds, outflows - X-rays: stars

\section{INTRODUCTION}

The Large Magellanic Cloud (LMC) supersoft X-ray source RX J0513.9-6951 (hereafter RX J0513) shows a prominent recurrency of optical high and low states with quasi-regular intervals $(\sim 120$ days high and $\sim 40$ days low states). Copious supersoft X-rays were detected only in the optical low states (e.g., Schaeidt et al. 1993). The transition mechanism between high and low states have not been fully elucidated yet, although a few ideas were proposed (see, e.g., Reinsch et al. 2000, for recent progress). We have found a new clue to this transition mechanism from modeling of the recurrent nova CI Aquilae.

CI Aql erupted in 2000 April has been densely observed in various optical bands (e.g., Hachisu, Kato, \& Schaefer 2002; Kiss et al. 2001; Lederle \& Kimeswenger 2002; Matsumoto et al. 2001; Schmeja, Armsdorfer, \& Kimeswenger 2000) and in X-ray bands (Greiner \& DiStefano 2002). It reached the optical maximum $(V \sim 9 \mathrm{mag})$ on 2000 May 5 . The visual brightness quickly decreased to $V \sim 13.5$ in about 50 days. Then, it stays at $V \sim 14$ for about 150 days, i.e., a plateau phase.

Matsumoto et al. (2001) reported a sharp $\sim 2$ mag drop of $R_{c}$-magnitude on 2000 November 23 , about 200 days after the optical maximum, and Kiyota (2001, VSNET archives, http://vsnet.kusastro.kyoto-u.ac.jp/vsnet/) also observed a $\sim 2$ mag drop of $I_{c}$-magnitude around the same day as Matsumoto et al.'s (2001) observation (see, e.g., Hachisu et al. 2002). Shortly after this drop, both the $R_{c^{-}}$and $I_{c^{-}}$-magnitudes recovered by $\sim 1$ mag but stayed at $\sim 1$ mag below the level before the drop. Hachisu \& Kato (2001a) and Hachisu et al. (2002) attributed this drop to cessation of massive winds by calculating time-evolution of optically thick winds on a $1.2 M_{\odot}$ white dwarf (WD).

The above feature of CI Aql light curve reminds us the optical high-to-low transition of RX J0513. In this paper, we try to reproduce the sharp drop in the optical light curves, both for CI Aql and RX J0513, by the same mechanism. In $\S 2$, our numerical method and models for the CI Aql 2000 outburst are briefly introduced and summarized. The numerical results for the CI Aql 2000 outburst are given in $\S 3$. We try to reproduce the transition between high and low states of RX J0513 in $\S 4$. Discussion follows in $\S 5$.

\section{BASIC MODEL OF CI AQUILAE 2000 OUTBURST}

The mid plateau phase is a common feature among the U Sco subclass of the recurrent nova class, to which both U Sco and CI Aql belong. The plateau phase is well explained by the contribution of an irradiated disk (e.g., Hachisu et al. 2000). The presence of the disk in outburst was observationally confirmed by Thoroughgood et al. (2001).

Our binary model is illustrated in Figure 1, which consists of a main-sequence star (MS) filling its Roche lobe, a WD photosphere, and a disk around the WD. A circular orbit is assumed. We also assume that the surfaces of the WD, the companion, and the disk emit photons as a blackbody at a local temperature which varies with position. We assume an axi-symmetric accretion disk with the size and thickness of

$$
R_{\mathrm{disk}}=\alpha R_{1}^{*},
$$

and

$$
h=\beta R_{\text {disk }}\left(\frac{\varpi}{R_{\text {disk }}}\right)^{\nu} \text {, }
$$


where $R_{\text {disk }}$ is the outer edge of the optically thick part of the accretion disk, $R_{1}^{*}$ is the effective radius of the inner critical Roche lobe for the WD component, $h$ is the height of the surface from the equatorial plane, and $\varpi$ is the distance from the symmetry axis. Here, we adopt $\nu=1$ during the strong wind phase, but $\nu=2$ when rapid mass accretion resumes after the optically thick wind stops, to mimic the flaring-up disk by spray (e.g., Schandl, MeyerHofmeister, \& Meyer 1997). The other two parameters of $\alpha$ and $\beta$ are determined by light curve fittings.

We have used the same parameters as those in Hachisu et al. (2002) unless otherwise specified. The mass of the white dwarf was determined to be $1.2 \pm 0.05 M_{\odot}$ by Hachisu \& Kato (2001a). The mass and temperature of the companion star were estimated to be $\sim 1.5 M_{\odot}$ and 7,300 K (see, e.g., Hachisu \& Kato 2001b, for the present evolutional status) from the light curve fitting of the orbital modulations in the 2000 outburst (Hachisu et al. 2002; Lederle \& Kimeswenger 2002). The inclination angle is a free parameter, which is redetermined from our light curve fitting.

Time evolution of the photospheric radius and temperature of the WD are calculated from Kato \& Hachisu's (1994) optically thick wind solutions (see Hachisu \& Kato 2001 b, for more details of the numerical methods). When the photospheric radius shrinks smaller than the binary size, the irradiation effects both of the disk and of the companion become important (see, e.g., Fig. 2 of Hachisu et al. 2000, for such an example).

\section{WHAT WE LEARNED FROM CI AQUILAE}

Orbital modulations during the plateau phase have been reported by Matsumoto et al. (2001). Their $R_{c}$ light curve data show a scatter of $\sim 0.1-0.2 \mathrm{mag}$ so that we average them into 36 phase bins as shown in Figure 2. No secondary eclipses were observed in contrast to the later phase orbital light curves (Lederle \& Kimeswenger 2002). To fit these orbital modulations as well as the entire evolution of $R_{c}$-magnitude, we have calculated light curves with various parameters ( $\alpha$ is changed by 0.1 steps, $\beta$ by 0.05 steps, and the inclination angle $i$ by $1^{\circ}$ steps). The best fit model is shown in Figure 2, i.e., $(\alpha, \beta)=(2.0,0.05)$ and $i=69^{\circ}$.

Here we plot two identical orbital modulations, but one is shifted by $0.36 \mathrm{mag}$ down. The brighter one corresponds to the earlier stage on 2000 July 17 while the fainter one does to that on 2000 October 1 . To reproduce the light curves with no secondary eclipses, we need both (1) large (area) irradiation effect to reproduce the brightness, and (2) bright sources other than the companion to eliminate the secondary eclipse. Then, we assumed that (a) the outer edge of an optically thick disk extends up to almost the orbital separation as illustrated in Figure 1a, and that (b) the WD photosphere is as large as $R_{\mathrm{WD}, \mathrm{ph}} \sim 0.35 R_{\odot}$ on July 17 and $R_{\mathrm{WD} \text {,ph }} \sim 0.15 R_{\odot}$ on October 1 if the other conditions are the same between these two epochs. Any light sources other than the disk or the companion are unlikely to contribute to the $R_{c}$-magnitude because the strong wind blows up off nebulosities around. This large extension of the disk naturally explains evident lack of secondary minima. The width of sharp eclipse minima, $\sim 0.05-0.1$ orbital phase, is caused by the partial occul- tation of the WD photosphere. These large photospheric radii $\left(R_{\mathrm{WD}, \mathrm{ph}} \sim 0.35 \rightarrow 0.15 R_{\odot}\right.$ from July 17 to October 1 ) are very consistent with our theoretical model because WDs blow a wind when $R_{\text {WD,ph }}>0.085 R_{\odot}$ (e.g., Kato \& Hachisu 1994; Hachisu \& Kato 2001b; Hachisu et al. 2002).

The effect of a larger inclination angle is canceled out by the effect of a larger radius of the disk. For example, a combination of the inclination angle $i=74^{\circ}$ and the disk of $(\alpha, \beta)=(3.0,0.05)$ can also reproduce the light curve modulations (dashed line) as shown in Figure 2. Its eclipse dip seems to be too deep to be compatible with the observation. However, the total amplitude of the modulations is $\sim 0.8 \mathrm{mag}$ (see Fig. 4 of Matsumoto et al. 2001, for the original observation) and still consistent. Here we adopted a set of $i=74^{\circ}$ and $(\alpha, \beta)=(3.0,0.05)$. In both $i=69^{\circ}$ and $i=74^{\circ}$ cases, the secondary occults only a part of the disk. This is, however, enough to make a orbital modulation observed because the central part of the disk is much brighter than the outer part.

The velocities of winds calculated are as fast as $1000-$ $1500 \mathrm{~km} \mathrm{~s}^{-1}$ (e.g., Kato \& Hachisu 1994) and the wind mass loss rate is as large as $10^{-6}-10^{-4} M_{\odot} \mathrm{yr}^{-1}$ (e.g., Hachisu \& Kato 2001b). Observationally much faster wind velocities are reported, for example, $2000-2500$ $\mathrm{km} \mathrm{s}^{-1}$ in CI Aql (see, e.g., Kiss et al. 2001). Such a strong wind affects the surface of the accretion disk. Because of a large velocity difference between the wind and the disk surface, it certainly drives the Kelvin-Helmholtz instability at the interface. Only the very surface layer of the disk is dragged away outward with the velocity at least several hundred $\mathrm{km} \mathrm{s}^{-1}$ like a free stream moving outward. This surface free stream is optically thick near the original disk but becomes optically thin somewhere outside because of geometrical dilution effect. We regard the transition place from optically thick to thin as the edge of the extended disk.

It should be noted that a high density part of the disk is hardly changed by this relatively tenuous surface free stream and still resides within its Roche lobe because the internal density of the disk is much denser than that of the WD wind. The wind mass loss rate is about $1 \times 10^{-6} M_{\odot} \mathrm{yr}^{-1}$ and its velocity is $\sim 1000 \mathrm{~km} \mathrm{~s}^{-1}$, so that the density of the wind is estimated from the continuity $\left(\dot{M}_{\text {wind }}=4 \pi r^{2} \rho v\right)$ to be about $10^{-11} \mathrm{~g} \mathrm{~cm}^{3}$ at the distance of $1 R_{\odot}$ from the center of the WD. On the other hand, the density of the standard accretion disk is about $1 \times 10^{-3} \mathrm{~g} \mathrm{~cm}^{3}$ at the same radius. Here, we assume the standard accretion disk model (Shakura \& Sunyaev 1973) with a mass accretion rate of $\sim 1 \times 10^{-7} M_{\odot} \mathrm{yr}^{-1}$.

After the wind stops, the photospheric radius of the WD quickly shrinks in the Kelvin-Helmholtz timescale of the envelope, i.e.,

$$
\tau_{\mathrm{KH}} \equiv \frac{\Delta E_{\mathrm{env}, \text { thermal }}}{L} \sim \frac{2.3 \times 10^{44} \mathrm{erg}}{2 \times 10^{38} \mathrm{erg} \mathrm{s}^{-1}} \sim 13 \text { days }
$$

where $\tau_{\mathrm{KH}}$ and $\Delta E_{\text {env,thermal }}$ are the Kelvin-Helmholtz timescale and the thermal energy of the WD envelope, respectively, and $L$ is the luminosity of the WD. Here, we adopt a typical thermal energy of the WD envelope from our solutions. It takes 20 days from $R_{\mathrm{WD}, \mathrm{ph}}=0.085 R_{\odot}$ to $0.04 R_{\odot}$ and takes another 20 days from $0.04 R_{\odot}$ to $0.02 R_{\odot}$ from our wind solutions and evolution models. 
The disk edge of optically thick part shrinks to a normal size of $0.7-0.8$ times the Roche lobe radius as observed in the supersoft X-ray sources (e.g., Hachisu et al. 2002; Lederle \& Kimeswenger 2002). This may occur in several dynamical timescales, i.e., a few to several days. Here, we adopt 10 days, i.e., the transition from configuration (a) to (b) in Figure 1. We adopt $(\alpha, \beta)=(0.8,0.05)$ for configuration (b). Then, the mass transfer rate from the MS to the WD increases to make a spray around the accretion disk as seen in luminous supersoft X-ray sources. This can be mimicked by a flaring edge of the accretion disk, i.e., configuration (c) in Figure 1 (see, e.g., Hachisu et al. 2002; Lederle \& Kimeswenger 2002, for light curves and flaring-up disk models after the wind stops). We adopt $(\alpha, \beta)=(0.8,0.3)$. It also takes several dynamical timescales. Here we take 10-20 days for the formation time of the spray. Thus, we are able to reproduce a sharp $\sim 2$ mag drop around 2000 November 23 and the ensuing $\sim 1$ mag recovery soon after the drop, as shown in Figure 3. A similar transition was observed in the U Sco 1999 outburst on HJD 2,451,244 just before the detection of supersoft X-rays (see Fig. 2 of Hachisu et al. 2000; Kahabka et al. 1999).

\section{LMC SUPERSOFT X-RAY SOURCE RX J0513.9-6951}

The LMC supersoft X-ray source RX J0513.9-6951 shows regular transitions between $60-120$ days optical high (X-ray off) and $30-40$ days optical low (X-ray on) state. The X-ray off/optical high state was understood by a large expansion of the WD photosphere, which is triggered by an increase in the mass transfer rate onto the WD (e.g., Meyer-Hofmeister et al. 1997; Pakull et al. 1993; Reinsch et al. 1996, 2000; Southwell et al. 1996). When the WD envelope expands, an optically thick wind begins to blow. As we already know in CI Aql, the sharp drop of $V$-mag is naturally explained by cessation of the wind. Our scenario is as follows:

1. We assume the metallicity of $Z=0.004$ and hydrogen content of $X=0.7$ for the envelope of the WD in RX J0513 since the LMC metallicity is reported to be about a third of the solar metallicity.

2. We have directly calculated the duration of the wind phase and the Kelvin-Helmholtz timescale on massive WDs of $1.0,1.1,1.2,1.3,1.35,1.36,1.37$, and $1.377 M_{\odot}$ (see also Hachisu \& Kato 2001b, for wind solutions). The $1.3 M_{\odot} \mathrm{WD}$ model gives us a reasonable wind timescale of $\tau_{\text {wind }} \sim 120$ days and a Kelvin-Helmholtz timescale of $\tau_{\mathrm{KH}} \sim 13$ days.

3. We use the ephemeris revised by Cowley et al. (2002). The inclination angle is not known but suggested to be low. Here we adopt $i=10^{\circ}$. The light curve is not so different among $i=0^{\circ}-20^{\circ}$. The calculated light curve is plotted in Figure 4, which is made by connecting brightness at the orbital phase 0.4 (phase 0.0 is the minimum of the light curve modulation). The mass ratio of $q=M_{\mathrm{MS}} / M_{\mathrm{WD}}=2$ is assumed because a high mass transfer rate of $\gtrsim 10^{-6} M_{\odot} \mathrm{yr}^{-1}$ is suggested (e.g. Southwell et al. 1996). The non-irradiated surface temperature of the lobe-filling MS companion is assumed to be 13,000 K (e.g., Fagotto et al. 1994).

4. We assume the original mass transfer rate of $2 \times$ $10^{-6} M_{\odot} \mathrm{yr}^{-1}$ as a reasonable value of thermally unsta- ble mass transfer for $q=2$ and $M_{\mathrm{MS}}=2.6 M_{\odot}$. This value is realized whenever the wind does not suppress the mass transfer. So, we start a rapid mass accretion to the WD at the rate of $2 \times 10^{-6} M_{\odot} \mathrm{yr}^{-1}$ (denoted by $\square$-mark in Fig. 4). The WD envelope gradually expands and eventually blows a wind (see the mid and bottom panels of Fig. 4). This causes a quick rise of $V$-mag. Here we assume the disk expansion of $(\alpha, \beta)=(3.0,0.05)$ in the wind phase. It takes a few to several dynamical timescales. We adopt 3 days. The massive winds having the rate of $\dot{M}_{\text {wind }} \sim 1 \times 10^{-8}-1 \times 10^{-6} M_{\odot} \mathrm{yr}^{-1}$ certainly absorb supersoft X-rays (e.g., Southwell et al. 1996).

5. This rapid mass accretion is suppressed by the strong wind, because the wind hits and then strips-off the very surface layer of the MS companion (see Hachisu et al. 1999a, for a description of the stripping effect by winds). We stop the rapid mass accretion when the wind mass loss rate increases and the mass-stripping rate overcomes the mass transfer rate of $2 \times 10^{-6} M_{\odot} \mathrm{yr}^{-1}$. This happens when the WD photosphere expands to the value shown in Figure $4(\triangle$-mark $)$. However, we still keep a low rate $\left(\sim 1 \times 10^{-7} M_{\odot} \mathrm{yr}^{-1}\right)$ during the period of draining of the disk (a few times viscous timescale of the disk $\sim 40-60$ days, e.g., Reinsch et al. 2000) even after the rapid mass accretion stops.

6. When the optically thick winds stops $(\bigcirc$-mark), we expect copious supersoft X-rays as observed in the $\mathrm{U}$ Sco 1999 outburst (Kahabka et al. 1999). Then the photosphere of the WD shrinks from $0.087 R_{\odot}\left(T_{\mathrm{ph}}=\right.$ $280,000 \mathrm{~K})$ to $0.038 R_{\odot}(430,000 \mathrm{~K})$ in 20 days. The photospheric temperature is consistent with the blackbody fitted temperatures $(30-40 \mathrm{eV})$ of the supersoft X-rays (e.g., Schaeidt et al. 1993) as shown in Figure 4. The disk shrinks to a normal size of $(\alpha, \beta)=(0.8,0.05)$ in a week and then the edge of the disk begins to flare up, i.e., $(\alpha, \beta)=(0.8,0.1)$ in another week, as described in $\S 3$.

7. Rapid mass accretion to the WD resumes ( $\square$-mark) $\sim 20$ days after the wind stops. We expect that the mass transfer rate gradually increases just after the wind stops but it takes $10-20$ days to reach the WD surface. This timescale may be regarded as a viscous timescale of the accretion disk (Reinsch et al. 2000). Then, the photospheric radius of the white dwarf expands in 20 days and eventually blows winds. Then, go to 4 and repeat the cycle.

Here we assume the apparent distance modulus of $(m-M)_{V}=18.25$ (intrinsic) $+0.4($ absorption $)=18.65$ to RX J0513. The calculated color of $(B-V)_{c}=$ -0.26 is roughly consistent with the observational color of $(B-V)_{o} \sim-0.14 \pm 0.04$ (Pakull et al. 1993), $(B-V)_{o} \sim-0.112 \pm 0.012$ (Cowley et al. 1993), or $(B-V)_{o} \sim-0.04$ (Cowley et al. 2002) for the color excess of $E(B-V) \sim 0.13$ (Gänsicke et al. 1998).

\section{DISCUSSION}

Thus, we are able to reproduce the transition between the optical high and low states of RX J0513 as shown in Figure 4. Our new model is essentially different from the previous expansion/contraction models of the white dwarf photosphere in the following sense: The emergence/decay timescale of the observed supersoft X-rays is as short as one or two days (Reinsch et al. 2000). 
This short timescale cannot be explained by the expansion/contraction of the envelope. We have calculated the timescales of photospheric contraction based on our WD envelope model (Kato \& Hachisu 1994) and show them in Figure 5 against the WD mass. The contraction timescale depends on the envelope mass of the WD and it is shortest just after the optically thick wind stops as shown in Figure 4. In this phase, the timescale of contraction by a factor of four is about three times the Kelvin-Helmholtz timescale. It is clear that the timescale of contraction by a factor of four cannot be shorter than six days even if the WD mass is very close to the Chandrasekhar mass. We may conclude that only the expansion/contraction model of the white dwarf photosphere cannot reproduce a very short timescale of X-ray on/off. We need another mechanism.

On the contrary, optically thick winds completely obscure supersoft X-rays within one day after it starts. The wind mass loss rate increases from zero to $\sim 2 \times$ $10^{-8} M_{\odot} \mathrm{yr}^{-1}$ in a day when it starts, or it decreases from $\sim 1 \times 10^{-8} M_{\odot} \mathrm{yr}^{-1}$ to zero in a day when it ceases, as easily seen from Figure 4 . This wind mass loss rate of $1 \times 10^{-8} M_{\odot} \mathrm{yr}^{-1}$ is large enough to absorb supersoft X- rays (see, e.g., discussion of Southwell et al. 1996).

In the scenario proposed by Hachisu et al. (1999a,b), all the progenitor systems of Type Ia supernovae once experience the optically thick wind phase during their way to Type Ia supernova. RX J0513 may be an example for such a wind phase. We have calculated the efficiency of mass accretion to be about $35 \%$ for the case of Figure 4 . The other $65 \%$ is blown in the wind or stripped off from the companion surface and eventually lost from the binary system. Assuming this efficiency being kept, we can expect that the white dwarf in RX J0513.9-6951 will explode as a Type Ia supernova after about $0.6 M_{\odot}$ is transferred even when the WD mass is $1.2 M_{\odot}$.

We thank K. Matsumoto for providing us machine readable data sets of the CI Aql 2000 outburst. We are also greatly indebted to F. Meyer and Emi Meyer-Hofmeister for stimulating us to work on RX J0513.9-6951 during our stay at the Max-Planck Institute for Astrophysics. This research has been supported in part by the Grant-in-Aid for Scientific Research (11640226) of the Japan Society for the Promotion of Science.

\section{REFERENCES}

Alcock, C. et al. 1996, MNRAS, 280, L49

Cowley, A.P., Schmidtke, P.C., Crampton, D., \& Hutchings, J.B. 2002, AJ, 124, 2233

Cowley, A. P., Schmidtke, P. C., Hutchings, J. B., Crampton, D., \& McGrath, T. K. 1993, ApJ, 418, L63

Fagotto, F., Bressan A., Bertelli G., Chiosi C. 1994, A\&AS, 105, 29

Gänsicke, B. T., van Teeseling, A., Beuermann, K., \& de Martino, D. 1998, A\&A, 333, 163

Greiner, J., \& DiStefano, R. 2002, ApJ, in press (astro-ph/0210035)

Hachisu, I., \& Kato, M. 2001a, ApJ, 553, L161

Hachisu, I., \& Kato, M. 2001b, ApJ, 558, 323

Hachisu, I., Kato, M., Kato, T., \& Matsumoto, K. 2000, ApJ, 528, L97

Hachisu, I., Kato, M., \& Nomoto, K. 1999a, ApJ, 522, 487

Hachisu, I., Kato, M., Nomoto, K., \& Umeda, H. 1999b, ApJ, 519 , 314

Hachisu, I., Kato, M., \& Schaefer, B. E. 2002, ApJ, in press (astro$\mathrm{ph} / 0210592)$

Kahabka, P., Hartmann, H. W., Parmar, A. N., \& Negueruela, I. 1999, A\&A, 374, L43

Kato, M., \& Hachisu, I., 1994, ApJ, 437, 802

Kiss, L. L., Thomson, J. R., Ogloza, W., Fürész, G., \& Sziládi, K. 2001, A\&A, 366, 858
Lederle, C., \& Kimeswenger, S. 2002, A\&A, in press (astro$\mathrm{ph} / 0209580)$

Matsumoto, K., Ishioka, R., Uemura, M., Kato, T., Kawabata, T. 2002, MNRAS, in press (astro-ph/0211241)

Matsumoto, K., et al. 2001, A\&A, 378, 487

Meyer-Hofmeister, E., Schandl, S., \& Meyer, F. 1997, A\&A, 321, 245

Nomoto, K., Thielemann, F., \& Yokoi, K. 1984, ApJ, 286, 644

Pakull, M. W., Motch, C., Bianchi, L., Thomas, H.-C., Guibert, J., Beaulieu, J. P., Grison, P., \& Schaeidt, S. 1993, A\&A, 278, L39

Reinsch, K., van Teeseling, A., Beuermann, K., \& Abbott, T. M. C. 1996, A\&A, 309, L11

Reinsch, K., van Teeseling, A., King, A. R., \& Beuermann, K. 2000, A\&A, 354, L37

Schaeidt, S., Hasinger, G., \& Truemper, J. 1993, A\&A, 270, L9

Schandl, S., Meyer-Hofmeister, E., \& Meyer, F. 1997, A\&A, 318, 73

Schmeja, S., Armsdorfer, B., \& Kimeswenger, S. 2000, Inf. Bull. Variable Stars, 4957

Shakura, N. I., \& Sunyaev, R. A. 1973, A\&A, 24, 337

Southwell, K. A., Livio, M., Charles, P. A., O'Donoghue, D., \& Sutherland, W. J. 1996, ApJ, 470, 1065

Thoroughgood, T. D., Dhillon, V. S., Littlefair, S. P., Marsh, T. R., \& Smith, D. A. 2001, MNRAS, 327, 1323 


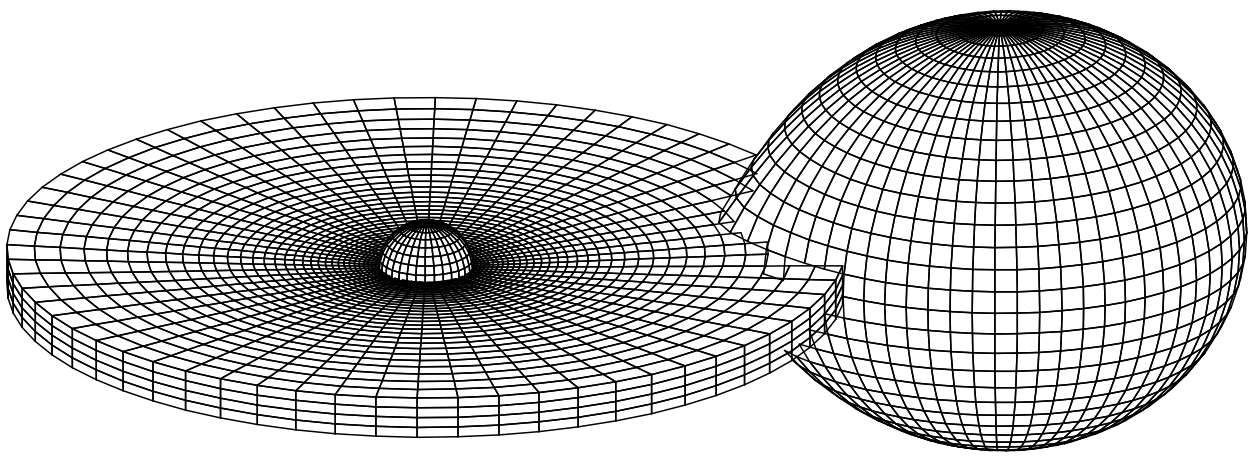

(a) optically thick wind phase
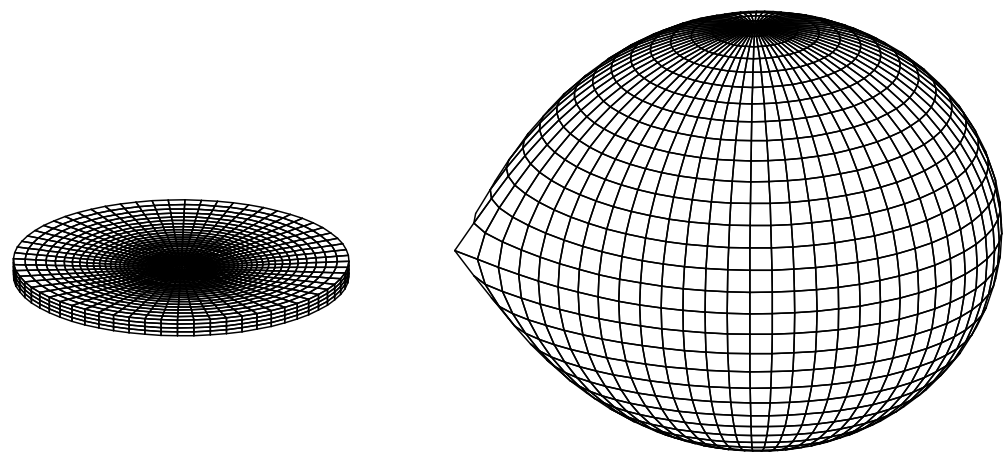

(b) just after the wind stops
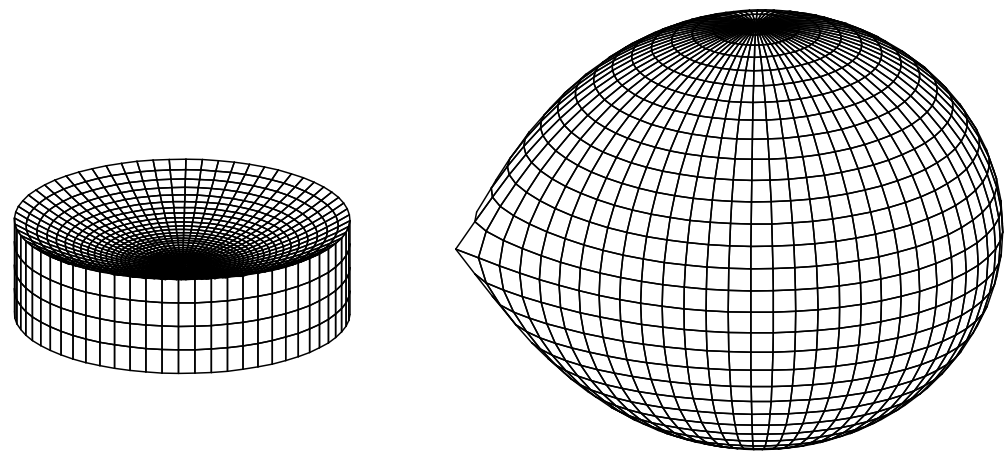

(c) mass accretion makes a spray

FIG. 1.- Configurations of our CI Aql model are illustrated: (a) in the massive wind phase (X-ray off), (b) just after the wind stops (X-ray on), and (c) during a rapid mass accretion phase soon after the wind stops (X-ray on). The cool component (right) is a slightly evolved MS companion $\left(1.5 M_{\odot}\right)$ filling its inner critical Roche lobe. The north and south polar areas of the cool component are irradiated by the hot component $\left(1.2 M_{\odot}\right.$ white dwarf, left). The separation is $a=4.25 R_{\odot}$; the effective radii of the inner critical Roche lobes are $R_{1}^{*}=1.53 R_{\odot}$, and $R_{2}^{*}=R_{2}=1.69 R_{\odot}$, for the primary white dwarf and the secondary main-sequence companion, respectively. (a) In our model, we regard the outer edge of the disk as the transition place from optically thick to thin. When the disk surface flow collides with the companion star, it may run around the companion. We approximate this situation simply by the disk configuration as shown in the figure. This round flow hardly contributes to light curves or line profiles because its irradiation effect is much smaller than the other parts the disk. (b) The disk shrinks to a normal size $(0.7-0.8$ times the Roche lobe size) in several orbital periods after the wind stops. (c) A rapid mass accretion resumes and makes a spray around the disk edge. 
Hachisu and Kato

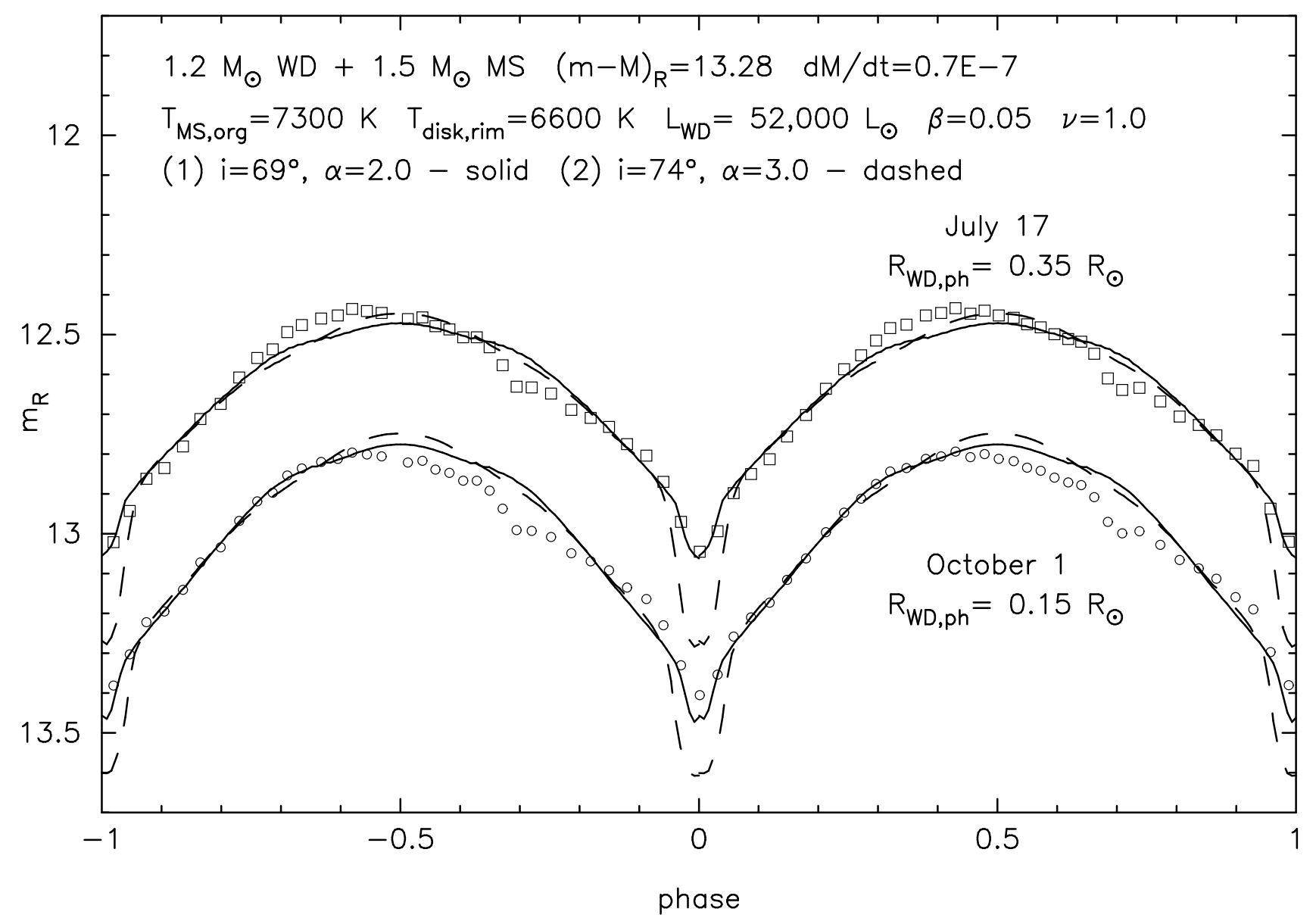

Fig. 2.- Calculated $R_{c}$ light curves are plotted against the binary phase (binary phase is repeated twice from -1.0 to 1.0 ) together with the observational points (Matsumoto et al. 2001) of the two epochs (open squares correspond to the brightness on 2000 July 17 and open circles are the brightness on 2000 October 1 ). Solid lines denote our calculated models with the inclination angle of $i=69^{\circ}$ but dashed lines are of $i=74^{\circ}$. Various system parameters are shown in the figure together with the best fitted parameters. 


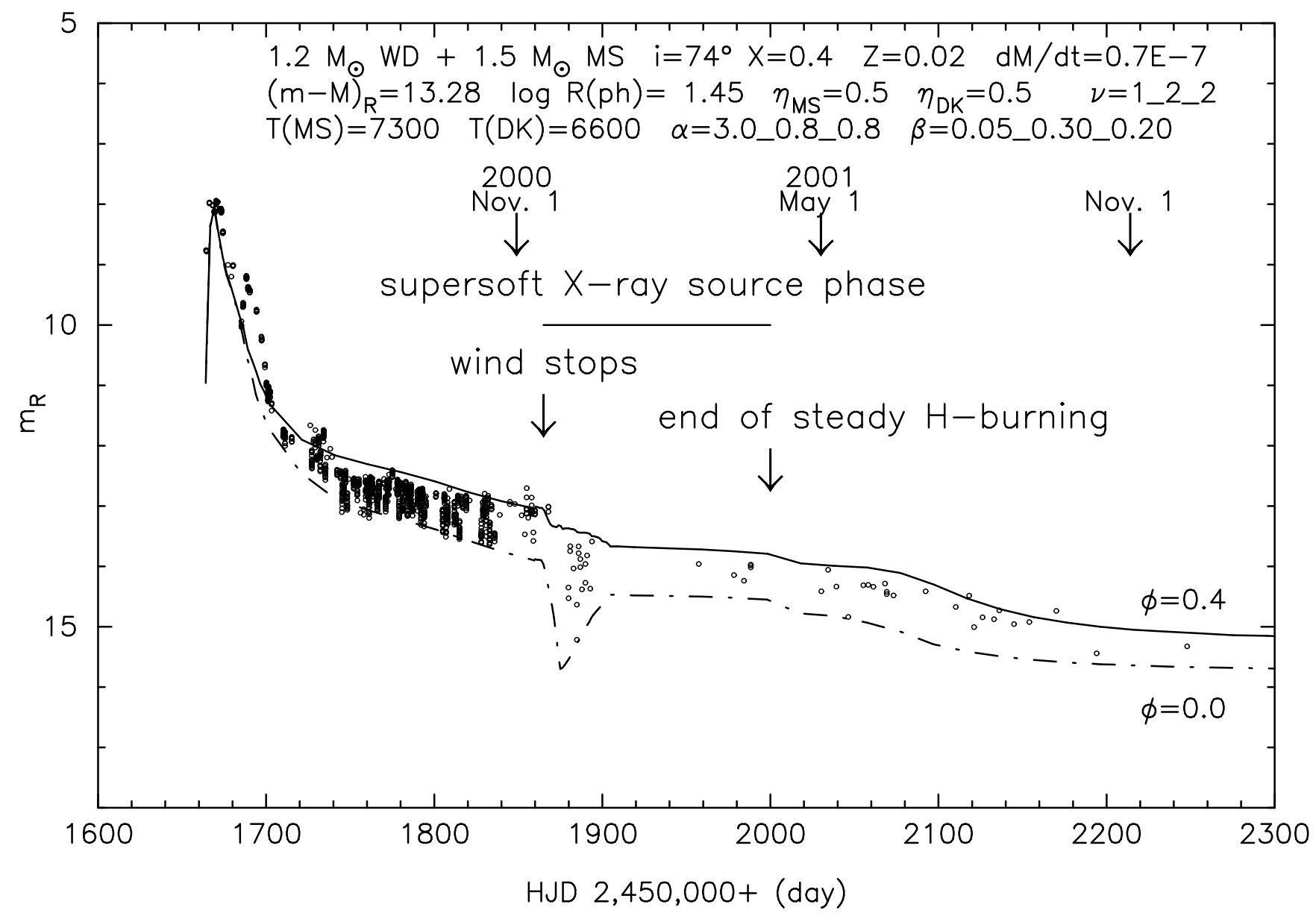

FIG. 3.- A calculated $R_{c}$-light curve are plotted against time (HJD 2,450,000+) together with the observational points of the CI Aql 2000 outburst (taken from Matsumoto et al. 2001, 2002). Small open circles indicate observational $R_{c}$-magnitudes. The light curve connects the brightness at binary phase 0.4 (solid), which is roughly corresponding to the upper bound of the light curve, or at mid eclipse (binary phase 0.0) (dashed), which gives us the lower bound. The apparent distance modulus of $(m-M)_{R}=13.28$ is used. The other system parameters are shown in the figure together with the best fitted parameters. Here we adopt the hydrogen content of $X=0.4$ for refinement instead of $X=0.35$ by Hachisu et al. (2002). 

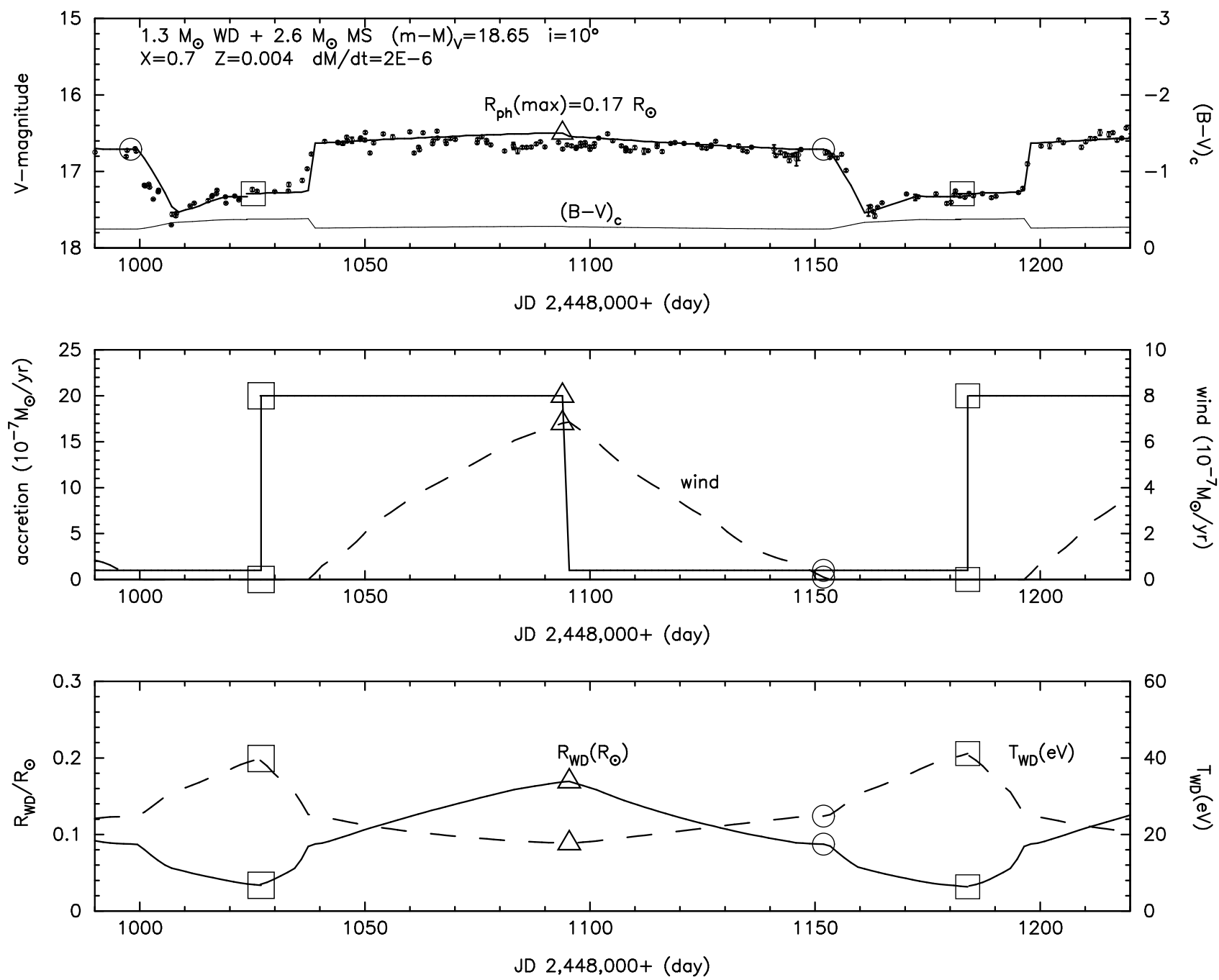

FIG. 4.- Top panel: $V$-magnitude light curves of RX J0513.9-6951 are plotted against time (JD 2,448,000+), together with the calculated color $(B-V)_{c}$ (lower portion). Small open circles are observational data with an error bar (Alcock et al. 1996). Thick solid lines correspond to our calculated light curve, connecting the brightness at orbital phase 0.4 . Thin solid lines denote the calculated color $(B-V)_{c}$. Starting a rapid mass accretion of $2 \times 10^{-6} M_{\odot} \mathrm{yr}^{-1}$ to the WD at epoch of open square, we continue it until epoch of open triangle. Optically thick winds stop at epoch of large open circle. The maximum expansion of the WD is reached at epoch of open triangle, the radius of which is shown above the open triangle. Mid panel: The accretion rate to the WD (solid line) and the wind mass loss rate from the WD (dashed line) are plotted against time. The other symbols have the same meaning as those in the top panel. Third panel: The white dwarf radius (solid line) and photospheric temperature (dashed line) are plotted against time. The other symbols are the same as those in the top panel. 


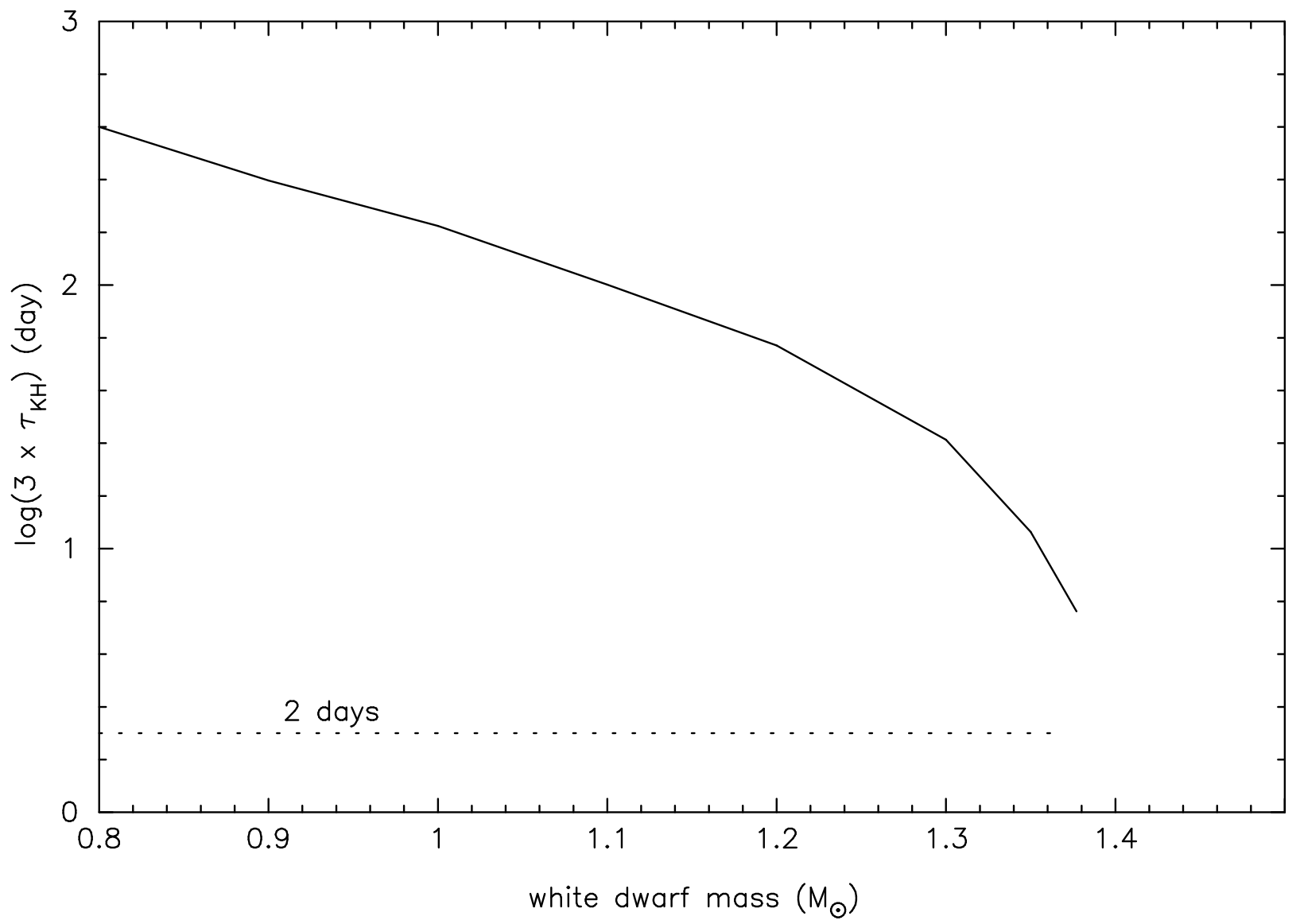

FIG. 5. - Timescale of contraction/expansion of white dwarf envelope with $Z=0.004$ and $X=0.7$ is plotted against the white dwarf mass. Here, we adopt time during which the white dwarf photosphere shrinks from $0.08 R_{\odot}$ to $0.02 R_{\odot}$ by a factor of four, which is about three times the Kelvin-Helmholtz timescale of the white dwarf envelope. 